\title{
Ueber den Hydroxylharnstoff;
} von Dr. W. F. C. Dresler und Dr. R. Stein*).

Durch Einwirkung von Hydroxylamin auf Cyansãure entsteht ein Körper $\mathrm{N}_{\mathbf{y}} \mathrm{CH}_{4} \mathrm{O}_{2}$, der seiner Bildung und Zusammenselzung nach als Hydroxylharnstoff zu betrachten ist. Die Darstellung desselben ist im Princip nicht verschieden von derjenigen anderer substiluirter Harnstoffe, bei der Ausführung sind indessen einige Schwierigkeilen durch besondere Vorsichtsmalsregeln zu überwinden.

Mischt man concentrirte Lösungen von Hydroxylaminsulfat und Kaliumcyanat (nach der bekannten Methode von Wurtz dargestellt), so tritt sofort starke Erwärmung und damit verbundene Ammoniakbildung und Gasentwickelung ein. Bei den ersten Versuchen wurde das gebildete Kaliumsulfat durch absoluten Alkohol ausgefällt und die alkoholische Lōsung vorsichtig verdunstet. Es blieb ein krystallinischer Rückstand, der meist vorzugsweise aus gewöhnlichem Harnstoff bestand, neben welchem wechselnde Quantitäten verschiedener anderer Körper zu erkennen waren; nur einmal gelang es, einen dieser letzteren, der unten beschrieben werden soll, rein darzustellen; Hydroxylharnstoff konnte nicht isolirt werden.

Es wurden darauf wieder äquivalente Mengen von $\mathrm{Hy}-$ droxylaminsulfat und Kaliumeyanat getrennt von einander in mōglichst wenig Wasser gelōst, die Erwärmung beim Mischen durch Abkühlen mit Schneewasser und sehr allmäliges Zu-

*) IIerr Dr. Dresler und Herr Dr. Ste in haben getrennt ron einander gearbeitet; die gegenseitige Ergïnzung der erhaltenen Rosultate liefs cine gemeinsame Mittheilung geeigneter crscheincn.

W. Lossen. 
sammengiefsen möglichst vermieden, dann sofort absoluter Alkohol zugesetzt, vom Kaliumsulfat abfiltrirt, und die Lösung mit absolutem Aether versetzt. Dadurch schied sich zunächst eine syrupartige Schichte ab, welche von der ätherischen Lösung getrennt, dann wiederholt in absolutem Alkohol gelöst und wieder mit Acther gefällt wurde; als ein erneuerter Zusatz von Aether keine Flüssigkeit mehr abschied, sondern nur noch eine Trübung bewirkte, die sich allmälig zu Krystallen vereinigte, wurden alle ätherischen Lösungen zusammengegossen, mit noch etwas Aether versetzt, nach dem Klarwerden der Flüssigkeit von dem ausgeschiedenen Krystallpulver (a) getrennt, und im Wasserbad bei möglichst niedriger Temperalur auf ein kleines Volumen verdampft. Beim Erkalten schied sich ein grau gefärbtes Krystallpulver ab, welches mit etwas absolutem Alkohol gewaschen, dann aus heifsem absolutem Alkohol umkrystallisirt wurde. Beim Erkalten schieden sich kleine Rosettchen ab (b); die Multerlauge lieferte eine zweite, etwas weniger reine Krystallisation. Sowohl das Krystellpulver a, als die Krystalle b erwiesen sich bei der Analyse als ziemlich reiner Hydroxylharnstoff :

Krystallpulver a :

1) 0,2692 Grm. gaben 0,1650 Kohlenstinre und 0,1438 Wasser.

2) 0,2858 Grm. gaben 94 CC. Stickgas, feucht gemessen bei $22^{\circ}$ und $756^{\mathrm{mm}}$ Druck.

3) Das Krystallpulver wurde nochmals in absolutem Alkohol gelüst, aus dieser Lösung durch Aether gefallt : 0,1885 Grm. gaben 0,1159 Kohlensilure und 0,0972 Wasser.

Krystalle b :

4) 0,1966 Grm. gaben 0,1182 Kohlensilure und 0,0945 Wasser.

5) 0,2292 Grin. (zweite Krystallisation) gaben 0,1397 Kohlensibure und 0,1198 Wasser.

6) 0,2741 Grm. (zweite Krystallisation) gaben 89 CC. Stickgas, feucht gemessen bei $19^{\circ}, 5$ und $749,5^{\mathrm{mm}}$ Druck. 


\begin{tabular}{|c|c|c|}
\hline \multicolumn{3}{|c|}{$\begin{array}{c}\text { Berechnet für } \\
\mathrm{N}_{2} \mathrm{CH}_{4} \mathrm{O}_{3}\end{array}$} \\
\hline $\mathbf{N}_{2}$ & 28 & 36,84 \\
\hline $\mathbf{C}$ & 12 & 15,79 \\
\hline $\mathbf{H}_{4}$ & 4 & 5,26 \\
\hline \multirow[t]{2}{*}{$\mathrm{O}_{2}$} & 32 & 42,11 \\
\hline & 76 & 100,00 \\
\hline
\end{tabular}

\begin{tabular}{cccc} 
1 & \multicolumn{4}{c}{ Drcsier } \\
37,0 & - & -5 ) & .u. 6) \\
16,72 & 16,77 & 16,39 & 16,62 \\
5,94 & 5,73 & 5,34 & 5,81 \\
- & - & - & -
\end{tabular}

Der bei sãmmtlichen Analysen etwas zu hoch gefundene Kohlenstoff beweist, dafs der Hydroxylharnstoff nicht ganz rein war.

Die im Vorgehenden beschriebene Methode lieferte eine im Verhältnifs zum angewandien Hydroxylaminsalz sehr geringe Ausbeute an Hydroxylharnstoff. Aufserdem erwies dieselbe sich als wenig zuverlässig, indem bei einer möglichst genauen Wiederholung der Darstellung die Isolirung des elwa gebildeten Hydroxylharnstolls von den reichlich vorhandenen Nebenproducten (meistentheils Harnstoff) nicht einmal gelang.

Es wurde daher anstalt des schwefelsauren das salpetersaure Hydroxylamin angewandt, welches in absolutem Alkohol leicht löslich ist. Es ist nicht nothwendig, dieses äufserst zerfliefsliche und sehr schwer krystallisirende Salz krystallisirt darzustellen, sondern man zersetzt die dem anzuwendenden Kaliumcyanat äquivalente Menge Hydroxylaminsulfat mit Baryumnitrat, dampft die Lōsung des Hydroxylaminnitrals Anfangs auf dem Wasserbad, schliefslich bei sehr gelinder Temperatur bis zum Syrup ein, indem man darauf achtet, dafs keine, Zersetzung des Salzes anzeigende Gasentwickelung eintritt, löst den Syrup in der zwei bis dreifachen Menge völlig absoluten Alkohols, kühlt diese Lösung auf -10 bis $-15^{\circ}$ ab und selzt dann die Lösung des Kaliumcyanats in möglichst wenig kaltem Wasser portionenweise zu. Sobald durch diesen Zusatz die Temperatur des Gemisches 
auf +5 bis $+10^{\circ}$ steigt, hält man mit dem Zusetzen der Kaliumcyanallōsung inne und setzt dasselbe erst fort, nachdem das Gemisch wieder auf etwa $-10^{\circ}$ abgekūhlt ist. So gelingt es, die Lösungen des Hydroxylaminsalzes und Kaliumcyanats zu mischen, ohne dafs eine erhebliche Gas- oder Ammoniakentwickelung eintritt. Eine Probe der vom ausgeschiedenen Salpeter möglichst schnell abfiltrirten Lösung wird mil absolutem Aether vermischt; scheidet sich dadurch eine wässerige Schicht ab, so ist vor dem Zusetzen von Aether zu der Hauplinenge der alkoholischen Lösung zu letzterer noch mehr absoluter Alkohol zuzusetzen, und zwar soviel, dafs durch Aether nur mehr ein krystallinischer Niederschlag (wesentlich Salpeter) entsteht. Dann erst wird die Hauptmenge des Aethers, etwa das 11/2fache Volum der alkoholischen Lōsung, zugesetzt, die nochmals filtrirto Lōsung auf dem Wasserbad bei gelinder Temperalur auf ein kleines Volum gebracht, und der beim Erkalten sich ausscheidende Hydroxylharnstoff aus Alkohol umkrystallisirt.

Das Abfiltriren der alkoholischen Lösung vom Salpeter und das Zusetzen von Aether mufs so rasch als möglich geschehen; in einem Fall, in welchem das letztere versăumt wurde, zeigte die anscheinend unzersetzt aus der Kältemiscliung herausgenommene alkoholische Lösung, nachdem sie eine Sommernacht hindurch gestanden halle, einen sehr starken Ammoniakgeruch, und der beim Verdunsten derselben über Schwefelsăure erhaltene Rückstand bestand fast nur aus gewöhnlichem Harnstoff, vermischt mit kleinen Mengen eines weiter unten $\mathrm{zu}$ beschreibenden Kaliumsalzes.

Bei der Analyse des aus salpetersaurem Hydroxylamin erhaltenen Hydroxylharnstoffs wurden folgendo Resultate erhalten :

$0,4995 \mathrm{Grm}$. gaben 0,2960 Kohlensilure and 0,2485 Wasser. 
0,4835 Grm. gaben 160 CC. Stickgas, feucht gemessen bei $24^{0}$ und $757,5^{\mathrm{min}}$ Druck.

\begin{tabular}{lrrr}
\multicolumn{2}{l}{ Bercehnet für } & $\mathrm{N}_{z} \mathrm{CH}_{4} \mathrm{O}_{2}$ & B te in \\
\hline $\mathrm{N}_{2}$ & 28 & 36,84 & 36,99 \\
$\mathrm{C}$ & 12 & 15,79 & 16,16 \\
$\mathrm{Ir}_{4}$ & 4 & 5,26 & 5,53 \\
$\mathrm{O}_{2}$ & 32 & 42,11 & - \\
\hline & 76 & $100,00$. &
\end{tabular}

Der Hydroxylharnstoff ist sehr leicht löslich in Wasser, deicht in heifsem, weniger leicht in kaltem Alkohol. Beim Erkalten heifs gesätligler alkoholischer Lösungen wurde or in kleinen Nadeln oder in Rosellchen erhalten, durch hinreichenden Zusatz von Aether zu der kalten concentrirten alkoholischen Lösung in mikroscopischen rhombischen Blättchen mit abgestumpften spitzen Winkeln. Die Lösungen sind indifferent gegen Lackuuspapier. Er schmilzt bei $128^{\circ}$ (S t c in), bei $130^{\circ}$ (Dresler); wird er in Schmelzen erhalten, so zersetzt er sich unter Gasentwickelung, welche allmälig sehr stürmisch wird; unter den entweichenden Gasen ist Kohlensăure und Ammoniak. Der nach Beendigung der Gasentwickelung bleibende Rückstand erstarrt bein Erkalten krystallinisch; er besteht wesentlich aus gewöhnlichem Harnstoff, vermischt mit anderen Substanzen, deren Menge zur Untersuchung zu gering war. Wird weiter erhitzt, so bilden sich natürlich die Zerselzungsproducte des gewölınlichen Harnstolls, wesentlich Cyanursäurc und Ammoniak. - Die Entstehung des gewölnnlichen llarnstoffs aus dem Hydroxylharnstoff ist wohl in der Weise zu crklären, dafs der Hydroxylharnstoff bei circa 130" zerfällt in Cyansäure und Hydroxylamin; lelzleres zersetzt sich gleichzeitig wesentlich in Stickstoff, Ammoniak und Wasser. Mit dem A sich Cyansãure zu Harnstofr, und zwar theils mit dem aus 
dem Hydroxylamin entstehenden Ammoniak, theils mit weileren Mengen, die durch Einwirkung des Wassers auf einen anderen Theil der Cyansãure entstehen.

Durch kochende Kalilauge wird der Hydroxylharnstoff unter Amnioniakentwickelung, durch rothe rauchende Salpetcrsãure unter starker Gasentwickelung zersetzt. - Seine Lōsungen zeigen ähnlichc reducirende Wirkungen wie dio Lösungen des Hydroxylamins. Eine mit Silbernitrat versetzte Lösung wird direct nicht gefältt, trübt sich jedoch nach kurzer Zeit durch ausgeschiedenes Silber; durch Erwārmen entstelt cin Silberspiegel, Ammoniakzusatz bewirkt ebenfalls sofort Reduction. - Quecksilberoxyd wird beim Kochen mit der Lösung des Hydroxylharnstoffs zu metallischem Quecksilber reducirt unter Gasentwickelung; die abfiltrirte Flüssigkeit hinterlăfst beim Verdunsten keinen Rückstand. - Kaliumbichromat wird ebenfalls reducirl, besonders leicht, wenn elwas Schwefelsăure zugesetzt wird. - Eine mit Kupfersalz und etwas Kalilauge versetzte Lösung scheidet Kupferoxydul ab. Kocht man überschüssiges Kupferoxyd mit Hydroxylaminlösung, so scheidet die heifs filtrirtc hellgrüne Lösung beim Erkalten einen hellgrünen flockigen Niederschlag ab. - Die Lösung des Hydroxylharnstoffs wird durch Eisenchlorid intensiv blanviolelt gefärbt; in wässeriger Lösung verschwindet die Färbung bald beim Stchen, rascher noch durch Kochen; in alkoholischer Lösung ist sie bleibend und bei Ueberschufs von Eisenchlorid fast schwarz oder in sehr verdünnten Lösungen dunkelgrün. Zusatz von Salz- oder Salpetersäure zerstört sofort alle Färbungen.

Salze des Hydroxylharnstofis zu erhalten, ist bis jetzt nicht gelungen. Als eine Auflōsung desselben in Salpetersãure bei gewōhnlicher Temperalur über Schwefelsäure verdunstet wurde, entwickelte die concentrirte Flüssigkeit Gas, und es blieb ein krystallinischer Rückstand, der wesentlich 
aus salpetersaurem Ammoniak besland. Eine ebenso verdunstete Lösung in Salzsăure hinterliefs ebenfalls einen krystallinischen Rückstand, aus dem durch Umkrystallisiren aus Alkohol ein völlig salzsäurefreies Product erhalten wurde, welches nicht mehr die für den Hydroxylharnstoff characteristische Färbung mit Eisenohlorid zeigte, einstweilen aber nocls nicht näher untersucht wurde.

Es wurde bereits oben erwähnt, dafs neben dem Hydroxylharnstoff und anstatt desselben andere Körper aus der Einwirkung von Hydroxylamin auf Cyansäure hervorgehen. Häufig tritt gewöhnlicher Harnstoff auf; von den noch nicht bekannlen oder nicht hinreichend erkannten Producten konnte wesentlich nur eines etwas näher untersucht werden. Dasselbe halte sich gebildet, als nicht sehr concentrirte Lõsungen von Kaliumcyanat und Hydroxylaminsulfat mit einander vermischt, das gebildete Kaliumsulfat durch Alkohol ausgefällt, die abfiltrirte alkoholische Lösung auf dem Wasserbad vorsichtig verdunstet und dann erst mit absolutem Aether versetzt worden war. Durch den Aetherzusuly schied sich eine wässerige, Harnstoff und nicht näher bestimmbare Producte enthaltende Schicht ab. Die ātherische Lösung wurde theilweise abdestillirt, dann übor Scliwefolsäure verdunstel; die concentrirte Lösung entwickelte etwas Gas und Ammoniak, scheint deinnach eine Zersetzung erlilfen zu huben, die möglicherweisc erst Veranlassung gab zur Bildung des schliefslich in Form von grünlich gefärbten Krystallen hinlerbleibenden Productes.

Die Krystalle wurden zwei Mal aus einem Gemisch von 4 Volumen absolutem Alkohol und 1 Volum Wasser umkrystallisirt und dadurch farblos erhalten.

Dic Analyse führte zur Formel $\mathrm{N}_{3} \mathrm{C}_{2} \mathrm{H}_{5} \mathrm{O}_{3}$. 
0,2723 Grm. gaben 0,204 Kohlensüure und 0,11 Wasser.

0,3163 Grm. gaben 99 CC. Stickgas, feucht gemegsen bei $20^{\circ}$ und $755^{\mathrm{mm}}$ Druck.

\begin{tabular}{rrrc}
\multicolumn{2}{l}{ Berechnet für } & \multicolumn{1}{c}{$\mathrm{N}_{3} \mathrm{C}_{2} \mathrm{H}_{8} \mathrm{O}_{3}$} & Dresler \\
\hline $\mathrm{N}_{8}$ & 42 & 35,29 & 35,5 \\
$\mathrm{C}_{8}$ & 24 & 20,17 & 20,43 \\
$\mathrm{II}_{6}$ & 5 & 4,20 & 4,49 \\
$\mathrm{O}_{3}$ & 48 & 40,34 & -
\end{tabular}

Die Entstehung dieses Körpers ist leicht verständlich; or setzt sich zusammen aus 2 Mol. Cyansâure und 1 Mol. Hydroxylamin, sei es nun, dafs diese sich direct mit einander vereinigen, oder dafs 2 Mol. Hydroxylharnstoff unter Ausscheidung von $1 \mathrm{Mol}$. Hydroxylamin zusammentreten :

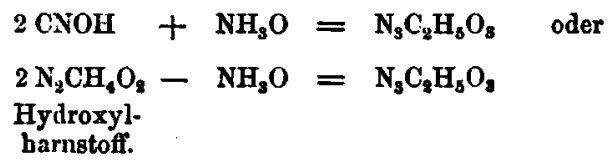

Bei der letzteren Annahme wird die Bildung der des Biurel's analog; ihrer empirischen Formel nach kann die Substanz $\mathrm{N}_{3} \mathrm{C}_{2} \mathrm{H}_{3} \mathrm{O}_{3}$ als Hydroxylbiuret angesehen werden. Ihre Reaclionen stellen sic ebenfalls neben andere Hydroxylaminderivate.

Der neue Körper ist löslich in Wasser, besonders leicht in heifsem; er löst sich in wasserhalligem Alkohol, in absolutem Alkohol dagegen selbst beim Kochen nur wenig. Ans alkoholischer Lösung wird er durch Zusatz von etwas Aether in mikroscopischen, vierseitigen, anscheinend schiefrhombischen Prismen erhalten. Gegen Lackmuspapier sind seine Lösungen indifferent. Er schmilzt bei $134^{\circ}$, zersetzt sich dann plötzlich unter ähnlichen Erscheinungen wie der Hydroxylharnstoff. Beim Erhitzen im Röhrchen bleibt schliefslich ein weifser, schwer sublimirbarer Körper. 
Kaliumhydrat und rothe rauchende Salpetersüure wirken ähnlich wie auf Hydroxylharnstoff. - Zusatz von Silbernitrat verändert die kalte Lösung Anfangs nicht; bei längerem Stehen aber oder beim Erwärmen scheidet sich ein weirser flockiger Niederschlag $a b$, der sich in Licht bald dunkel färbt. Der Nicderschlag ist in Ammoniak löslich; die ammoniakalische Lõsung wird in der Kälte allmälig, beim Erhitzen sofort reducirt, unter Bildung eines Mctallspiegels. Quecksilberoxyd und kalische Kupfurlösung worden beim Erwärmen reducirt. Wird cinc wässerige Lösung längere Zeit mit überschüssigem Iupferoxyd gekocht, dänn filtrirt, so scheiden sich aus der Lösung kleine undeutliche Kryställchen ab. Trägt man dagegen nicht überschüssiges Kupferoxyd in die Lösung ein und erhitzt nur kurze Zeit zum Sieden, so verbindet sich das Kupferoxyd mit dem Körper $\mathrm{N}_{3} \mathrm{C}_{2} \mathrm{H}_{3} \mathrm{O}_{3}$ zu hellgrünen mikroscopischen haarförmigen Nadeln. Eine Lösung von Eisenchlorid bringt keine Färbung hervor.

Bei dem Versuche, ein salzsaures Salz des neuen Körpers darzustellen, wurde dersclbe in verdünnter Salzsäure gelöst und die Lösung bei gewöhnlicher Temperalur über Schwefelsäure und Kaliumliydrat verdunstet. Es blieb ein krystallinischer Rückstand, der durch Umkrystallisiren aus absolutem Alkohol in rosetteartigen Krystallen erhalten wurde, die chlorfrei waren und sich durch die Analyse als Hydroxylharnstoff erwiesen :

0,2035 Grm. gaben 0,1218 Kohlensäuro und 0,0987 Wasser.

0,2004 Grm. gabeu 81 CC. Stickgas, feucht gemessen bei $19^{\circ}$ und $758,5^{\mathrm{mm}}$ Druck.

Diefs entspricht 16,32 pC. Kohlenstoff, 5,39 pC. Wasserstoff und 37,1 pC. Stickstoff, wilhrend dic Formel des Hydroxylharnstoffs $15,79 \mathrm{pC}$. Kohlenstoff, $5,26 \mathrm{pC}$. Wasserstoff und 36,84 pC. Stickstoff verlangt.

Der erhaltene Hydroxylharnstoff zeigte die characteristische blauviolette Färbung mit Eisenchlorid. 
Es hatte demnach durch die Einwirkung von Salzsăure eine Zersetzung in der Art stattgefunden, dars aus einem Mol. des Körpers $\mathrm{N}_{3} \mathrm{C}_{2} \mathrm{H}_{5} \mathrm{O}_{3}$ ein Mol. Cyansäure abgespalten wurde :

$$
\mathrm{N}_{2} \mathrm{C}_{8} \mathrm{H}_{8} \mathrm{O}_{8}-\mathrm{CNOH}=\mathrm{N}_{2} \mathrm{CH}_{4} \mathrm{O}_{8} .
$$

Ammoniak, welches bei Gegenwart von Wasser aus der ausiretenden Cyansãure entslehen mufste, war im Filtrat vom Hydroxylharnstoff in Form von Chlorammonium leicht nachzuw eisen.

Die erwähnte Bildung von Hydroxylharnstoff aus dem Körper $\mathrm{N}_{3} \mathrm{C}_{2} \mathrm{H}_{5} \mathrm{O}_{3}$ steht in einem scheinbaren Widerspruch mit der Zersetzbarkeit des Hydroxylharnstoffs durch Salzsăure. Allein es ist begreiflich, dafs die Reaction nur bis zur Abspaltung von ein Mol. Cyansäure fortgeschritten sein konnte, ohne dafs deshalb schon aller Hydroxylharnstoff zersetzt sein mufste.

Zum Sclilufs sei noch ein Kaliumsalz erwähnt, welches, wie bereits oben gesagt wurde, bei einem Versuch zur Darstellung des Hydroxylharnstoffs aus Hydroxylaminnitrat und Kaliumcyanat erhalten wurde. Dasselbe fand sich theils dem gewöhnlichen Harnstoff, der die Hauptmenge des anstatt des erwarteten Hydroxylharnstoffs erhaltenen Productes ausmachte, beigemengt, theils dem Salpeter, der durch Umsetzung des Hydroxylaminnitrats mit dem Kaliumcyanat entstand. Der Harnstoff wurde entfernt durch Auskochen mit möglichst wenig 90grädigem Alkohol, in welchem das Kaliumsalz sehr wenig löslich ist. Aus dem Salpeler wurde das Salz durch Auskochen desselben mit viel Weingeist ausgezogen, dann durch oft wiederholtes Umkrystallisiren aus Weingeist und durch Auflōsen in Wasser und Fãllen der wãsserigen Lösung durch absoluten Alkohol gereinigt, bis es keine Reaction auf Sal- 
252 Dresler u. Stein, ulber den Bydroxylharnstoff.

petersāure mehr zeigte. Die Analyse stimmt hinreichend mit der Formel $\mathrm{N}_{6} \mathrm{C}_{4} \mathrm{H}_{9} \mathrm{O}_{6} \mathrm{~K}$.

1) 0,2565 Grm. (mit chromsaurom Blei verbrannt) gaben 0,1675 Kohlensüure und 0,0855 Wassor.

2) 0,2845 Grm. gaben 77,5 CC. Stickgas, feucht gemesson bei $23^{\circ}$ und $757^{\mathrm{mm}}$ Druck.

3) 0,162 Grm. gaben 0,049 Kaliumsulfat.

4) 0,1185 Grm. gaben 0,037 Kaliumsulfat.

\begin{tabular}{|c|c|c|c|c|}
\hline & \multicolumn{2}{|c|}{ stern } \\
\hline & & & $\widehat{1,2 u .3)}$ & 4) \\
\hline $\mathbf{N}_{8}$ & Berechnet fuir $\underbrace{\mathrm{N}_{6} \mathrm{C}_{4} \mathrm{H}_{8} \mathrm{O}_{6} \mathrm{~K}}$ & 30,44 & 30,58 & - \\
\hline $\mathrm{C}_{4}$ & 48 & 17,39 & 17,81 & - \\
\hline $\mathbf{H}_{9}$ & 9 & 3,26 & 3,71 & - \\
\hline $\mathrm{O}_{6}$ & 96 & 34,78 & - & - \\
\hline \multirow[t]{2}{*}{$\mathbf{K}$} & 39,2 & 14,13 & 13,56 & 13,99 \\
\hline & 276,2 & 100,00 . & & \\
\hline
\end{tabular}

Das Salz bildet miskroscopische farblose Nadeln. Seine Lösungen färben Eisenchlorid intensiv kirschroth; die Färbung crhält sich auch in wässeriger Lösung, verschwindet aber durch einen Tropfen Mineralsăure.

Nach seiner Formel, die indefs einer weiteren Bestätigung noch bedarf, scheint dieses Sulz in einer einfachen Beziehung 2u dem Körper $\mathrm{N}_{3} \mathrm{C}_{2} \mathrm{H}_{5} \mathrm{O}_{3}$ zu stehen. Es leitet sich ab von 2 Mol. dieses Körpers, indem darin 1 At. Wasserstof durch 1 At. Kalium vertreten ist. Anderc Beobachtungen zeigen, dafs in den amidarligen Derivaten des Hydroxylamins Wasserstoff durch Metall ersetzbar ist; da nun die aus Cyansãure und Hydroxylamin entstehenden Körper Anide sind, die dus Radical CO enthalten, so erscheint auch in ihnen die Vertretbarkeit von Wasserstoff durch Metall möglich.

W. Lossen's Laboratorium in Heidelberg, 31. März 1869. 